Marquette University

e-Publications@Marquette

Electrical and Computer Engineering Faculty

Electrical and Computer Engineering, Department

Research and Publications

$1-1-2016$

\title{
Robust Multi-Criteria Optimal Fuzzy Control of Continuous-Time Nonlinear Systems
}

Xin Wang

Southern Illinois University Edwardsville

Edwin E. Yaz

Marquette University, edwin.yaz@marquette.edu

Published version. Systems Science \& Control Engineering, Vol. 4, No. 1 (2016): 59-67. DOI. (C) 2016 The Author(s). Used with permission. 


\title{
Robust multi-criteria optimal fuzzy control of continuous-time nonlinear systems
}

\author{
Xin Wang ${ }^{\mathrm{a}}$ and Edwin E. Yaz ${ }^{\mathrm{b}}$

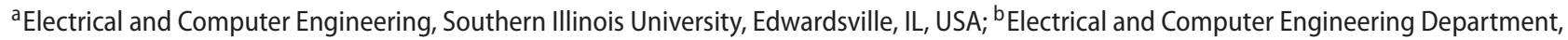 \\ Marquette University, Milwaukee, WI, USA
}

\begin{abstract}
This paper presents a novel fuzzy control design of continuous-time nonlinear systems with multiple performance criteria. The purpose behind this work is to improve the traditional fuzzy controller performance to satisfy several performance criteria simultaneously to secure quadratic optimality with inherent stability property together with dissipativity type of disturbance reduction. The Takagi-Sugeno fuzzy model is used in our control system design. By solving the linear matrix inequality at each time step, the control solution can be found to satisfy the mixed performance criteria. The effectiveness of the proposed technique is demonstrated by simulation of the control of the inverted pendulum system.
\end{abstract}

ARTICLE HISTORY

Received 1 March 2016 Accepted 28 April 2016

\section{KEYWORDS}

Fuzzy control; robust control linear matrix inequality

\section{Introduction}

Over the past two decades, fuzzy control systems have obtained growing popularity in nonlinear system control applications (Takagi \& Sugeno, 1985; Tanaka and Sugeno, 1990; Tanaka, Ikeda, and Wang, 1996; Tanaka \& Wang, 2001; Wang, 1994; Wang, Tanaka, \& Griffin, 1996). The Takagi-Sugeno (T-S) fuzzy model can effectively approximate a wide class of nonlinear systems. The T-S model approach decomposes the task of nonlinear system control into a group of local linear controls based on a set of design-specific model rules. It also provides a mechanism to blend all these local linear control problems together to achieve overall control of the original nonlinear system. In general, the T-S fuzzy model represents the nonlinear plant as an average of the weighted sum of a set of local linear systems. This particular representation provides a favourable form for the stability analysis and controller design by using the linear control techniques. In this regard, the T-S fuzzy control technique has a unique advantage over other kinds of nonlinear control techniques.

Recent research on fuzzy control system design aims to improve the optimality and robustness of the controller performance by combining the advantage of modern control theory with the T-S fuzzy model (Dong, Wang, \& Yang, 2009; Lam, Li, \& Liu, 2013). Based on the T-S fuzzy model framework, many systematic approaches for stability analysis, observer design, and control synthesis are studied in the literature. Particularly, the control synthesis based on quadratic Lyapunov function approaches has been extensively studied in Fang, Liu, Kau, Hong, \& Lee (2006); Kim \& Lee (2000); Liu \& Zhang (2003); Sala \& Arino (2007); Teixeira \& Zak (1999); Teixeira, Assuncao, \& Avellar (2003); and Tuan, Apkarian, Narikiyo, \& Yamamoto (2001). Since a common quadratic Lyapunov function is independent of fuzzy membership functions, the results based on a single Lyapunov function might be conservative. Therefore, in order to address this issue, piecewise Lyapunov functions (Feng, 2003; Johansson, Rantzer, \& Arzen, 1999), parameter-dependent Lyapunov functions (fuzzy Lyapunov functions) (Guerra \& Vermeiren, 2004; Tanaka, Hori, \& Wang, 2003; Wang \& Sun, 2005; Wang, Chen, \& Sun, 2007), and k-sample variation Lyapunov functions (Kruszewski, Wang, \& Guerra, 2008) have been proposed for less conservative results. In the aforementioned works, the parallel distributed compensation control scheme, that is, the controller shares the same fuzzy membership rules with the fuzzy model, is extensively applied for designing fuzzy controllers (Tanaka \& Wang, 2001).

Meanwhile, it is important to consider not only the stability, but also some control performance requirements, such as $H_{\infty}$ control performance and bounded cost constraints, which have also been extensively exploited in the recent literature. Among them, the linear matrix inequality (LMI)-based control design can be found in Lee, Jeung, \& Park (2001); Lo \& Lin (2004); Tanaka (2001); and Tseng \&

\section{CONTACT Xin Wang xwang@siue.edu}


Hwang (2007). The guaranteed cost control can be found in Chen, Liu, Tong, \& Lin (2007) and Wu (2004). The $H_{\infty}$ control with quadratic $D$ stability constraints can be found in Nguang \& Shi (2006).

Other analysis techniques and fuzzy controllers based on the T-S fuzzy model have also been studied. The circle criteria were studied to investigate the stability of the fuzzy model-based control systems in Lu, Huang, Gao, Ban, \& Yin (2007). Model reference approaches were developed so that the system states of the nonlinear model are driven to follow the stable reference model (Lam, Leung, \& Tam, 2001). Sliding mode control techniques were developed to analyse stability and controller synthesis in Lam, Leung, and Tam (2002). Adaptive fuzzy control schemes are proposed in Tong, He, and Zhang (2009) and Tong, Liu, and Li (2010), in which the parameters of a fuzzy controller are updated to stabilize the nonlinear system. The sampled data of fuzzy model-based systems and time-delayed fuzzy control system are investigated in Gao, Liu, and Lam (2009) and Lin, Wang, and Lee (2005, 2006).

The aforementioned work is based on certain given criteria. In order to provide a more flexible fuzzy modelbased controller design, we propose the robust multicriteria optimal fuzzy control design of continuous-time nonlinear systems in this paper. We characterize the solution of the nonlinear continuous-time control system with the LMI, which provides a sufficient condition for satisfying various performance criteria. A preliminary investigation into the LMI approach to nonlinear fuzzy control systems can be found in Takagi \& Sugeno (1985); Tanaka \& Sugeno (1990); and Wang (1994). The purpose behind this novel approach is to convert a nonlinear system control problem into a convex optimization problem which is solved by an LMI at each time step. The recent development in numerical techniques for convex optimization provides efficient algorithms for solving LMIs. If a solution can be expressed in an LMI form, then there exist optimization algorithms providing efficient global numerical solutions (Boyd, Ghaoui, Feron, \& Balakrishnan, 1994). Therefore if the LMI is feasible, then the LMI control technique provides globally stable solutions satisfying the corresponding mixed performance criteria at each time step (Huang \& Lu, 1996; Mohseni, Yaz, \& Olejniczak, 1998; Wang \& Yaz, 2010a, 2010b; Wang, Yaz, \& Jeong, 2010; Wang, Yaz \& Yaz, 2010, 2011). Moreover, we propose to employ the mixed performance criteria to design the controller, guaranteeing quadratic suboptimality with inherent stability property in combination with dissipativity type of disturbance attenuation.

The rest of the paper is organized as follows. In the following section, we first describe the T-S fuzzy model. We then introduce the mixed performance criteria in
Section 3. Then, the LMI control solution is derived to characterize the optimal and robust fuzzy control of nonlinear systems. Finally, the inverted pendulum on a cart control problem is used as an illustrative example. The following notation is used in this work: $x \in \Re^{n}$ denotes $n$ dimensional real vector with norm $\|x\|=\left(x^{\top} x\right)^{1 / 2}$, where $(\cdot)^{\top}$ indicates transpose. $A \geq 0$ for a symmetric matrix denotes a positive semi-definite matrix. $L_{2}$ is the space of infinite sequences of finite dimensional random vectors with finite energy: $\int_{0}^{\infty}\|x(t)\|^{2} \mathrm{~d} t<\infty$.

\section{T-S system model}

The importance of the T-S fuzzy system model is that it provides an effective way to decompose a complicated nonlinear system into local dynamical relations and express those local dynamics of each fuzzy implication rule by a linear system model. The overall fuzzy nonlinear system model is achieved by fuzzy 'blending' of the linear system models, so that the overall nonlinear control performance is achieved.

The ith rule of the T-S fuzzy model can be expressed by the following forms:

MODEL RULE $i$ :

IF $\varphi_{1}(t)$ is $M_{i 1}, \varphi_{2}(t)$ is $M_{i 2}, \ldots$, and $\varphi_{p}(t)$ is $M_{i p}$,

THEN, the input-affine continuous-time fuzzy system equation is:

$$
\left\{\begin{array}{l}
\dot{x}(t)=A_{i} x(t)+B_{i} u(t)+F_{i} w(t) \\
y(t)=C_{i} x(t)+D_{i} u(t)+Z_{i} w(t)
\end{array} \quad i=1,2,3, \ldots, r,\right.
$$

where $x(t) \in \Re^{n}$ is the state vector; $u(t) \in \Re^{m}$ is the control input vector; $y(t) \in \Re^{q}$ is the performance output vector; $w(t) \in \Re^{s}$ is the $L_{2}$ type of disturbance; $r$ is the total number of the model rules; $M_{i j}$ is the fuzzy set; $A_{i} \in$ $\Re^{n \times n}, B_{i} \in \Re^{n \times m}, F_{i} \in \Re^{n \times s}, C_{i} \in \Re^{q \times n}, D_{i} \in \Re^{q \times m}, Z_{i} \in$ $\Re^{q \times s}$ are the coefficient matrices; and $\varphi_{1}, \ldots, \varphi_{p}$ are the known premise variables which can be functions of state variables, external disturbance, and time.

It is assumed that the premises are not the function of the input vector $u(t)$, which is needed to avoid the defuzzification process of the fuzzy controller. If we use $\varphi(t)$ to denote the vector containing all the individual elements $\varphi_{1}(t), \ldots, \varphi_{p}(t)$, then the overall fuzzy system is

$$
\begin{aligned}
\dot{x}(t) & =\frac{\sum_{i=1}^{r} g_{i}(\varphi(t))\left(A_{i} x(t)+B_{i} u(t)+F_{i} w(t)\right)}{\sum_{i=1}^{r} g_{i}(\varphi(t))} \\
& =\sum_{i=1}^{r} h_{i}(\varphi(t))\left\{A_{i} x(t)+B_{i} u(t)+F_{i} w(t)\right\}, \\
y(t) & =\frac{\sum_{i=1}^{r} g_{i}(\varphi(t))\left\{C_{i} x(t)+D_{i} u(t)+Z_{i} w(t)\right\}}{\sum_{i=1}^{r} g_{i}(\varphi(t))} \\
& =\sum_{i=1}^{r} h_{i}(\varphi(t))\left\{C_{i} x(t)+D_{i} u(t)+Z_{i} w(t)\right\},
\end{aligned}
$$


where

$$
\begin{aligned}
\varphi(t) & =\left[\varphi_{1}(t), \varphi_{2}(t), \ldots, \varphi_{p}(t)\right], \\
g_{i}(\varphi(t)) & =\prod_{j=1}^{p} M_{i j}\left(\varphi_{j}(t)\right), \\
h_{i}(\varphi(t)) & =\frac{g_{i}(\varphi(t))}{\sum_{i=1}^{r} g_{i}(\varphi(t))}
\end{aligned}
$$

for all time $t$. The term $M_{i j}\left(\varphi_{j}(t)\right)$ is the grade membership of $\varphi_{j}(t)$ in $M_{i j}$.

Since

$$
\begin{aligned}
& \sum_{i=1}^{r} g_{i}(\varphi(t))>0 \\
& g_{i}(\varphi(t)) \geq 0, \quad i=1,2,3, \ldots, r
\end{aligned}
$$

we have

$$
\begin{aligned}
& \sum_{i=1}^{r} h_{i}(\varphi(t))=1, \\
& h_{i}(\varphi(t)) \geq 0, \quad i=1,2,3, \ldots, r
\end{aligned}
$$

for all time $t$.

It is assumed that the state is available for feedback and the nonlinear state feedback control input is given by

$$
u(t)=-\sum_{i=1}^{r} h_{i}(\varphi(t)) K_{i} x(t)
$$

Substituting this into the system and performance output equations, we have

$$
\begin{aligned}
\dot{x}(t)= & \sum_{i=1}^{r} \sum_{j=1}^{r} h_{i}(\varphi(t)) h_{j}(\varphi(t))\left\{A_{i}-B_{i} K_{j}\right\} x(t) \\
& +\sum_{i=1}^{r} h_{i}(\varphi(t)) F_{i} w(t), \\
y(t)= & \sum_{i=1}^{r} \sum_{j=1}^{r} h_{i}(\varphi(t)) h_{j}(\varphi(t))\left\{C_{i}-D_{i} K_{j}\right\} x(t) \\
& +\sum_{i=1}^{r} h_{i}(\varphi(t)) Z_{i} w(t) .
\end{aligned}
$$

Using the notation

$$
\begin{aligned}
& G_{i j}=A_{i}-B_{i} K_{j}, \\
& H_{i j}=C_{i}-D_{i} K_{j}
\end{aligned}
$$

then the system equation becomes

$$
\begin{aligned}
\dot{x}(t)= & \sum_{i=1}^{r} \sum_{j=1}^{r} h_{i}(\varphi(t)) h_{j}(\varphi(t)) \cdot G_{i j} \cdot x(t) \\
& +\sum_{i=1}^{r} h_{i}(\varphi(t)) F_{i} w(t),
\end{aligned}
$$

$$
\begin{aligned}
y(t)= & \sum_{i=1}^{r} \sum_{j=1}^{r} h_{i}(\varphi(t)) h_{j}(\varphi(t)) \cdot H_{i j} \cdot x(t) \\
& +\sum_{i=1}^{r} h_{i}(\varphi(t)) Z_{i} w(t) .
\end{aligned}
$$

\section{General performance criteria}

Consider the quadratic Lyapunov function

$$
V(t)=x^{\top}(t) P x(t)>0
$$

for the following differential inequality

$$
\begin{gathered}
\dot{V}(t)+x^{\top}(t) Q x(t)+u^{\top}(t) R u(t)+\alpha \cdot y^{\top}(t) y(t) \\
-\beta \cdot y^{\top}(t) w(t)+\gamma \cdot w^{\top}(t) w(t) \leq 0
\end{gathered}
$$

with $Q>0, R>0$ functions of $x$.

Note that upon integration over time from 0 to $T_{f}$, Equation (17) yields

$$
\begin{aligned}
& V\left(T_{f}\right)+\int_{0}^{T_{f}}\left[x^{\top}(t) Q x(t)+u^{\top}(t) R u(t)\right] \mathrm{d} t+\int_{0}^{T_{f}}[\alpha \\
& \left.\cdot y^{\top}(t) y(t)-\beta \cdot y^{\top}(t) w(t)+\gamma \cdot w^{\top}(t) w(t)\right] \mathrm{d} t \leq V(0)
\end{aligned}
$$

for all $T_{f}>0$.

By properly specifying the value of the weighing matrices $Q, R, C_{i}, D_{i}, Z_{i}$, and $\alpha, \beta, \gamma$, the mixed performance criteria can be used in nonlinear control design, which yields a mixed Nonlinear Quadratic Regulator (NLQR) (Wu $\&$ Cai, 2004) in combination with the dissipativity type performance index with disturbance reduction capability. For example, if we take $\alpha=1, \beta=0, \gamma<0$, Equation (18) yields

$$
\begin{aligned}
& V\left(T_{f}\right)+\int_{0}^{T_{f}}\left[x^{\top}(t) Q x(t)+u^{\top}(t) R u(t)+y^{\top}(t) y(t)\right] \mathrm{d} t \\
& \quad \leq V(0)-\gamma \cdot \int_{0}^{T_{f}}\left[w^{\top}(t) w(t)\right] \mathrm{d} t
\end{aligned}
$$

which is the mixed suboptimal NLQR- $H_{\infty}$ design (Wang \& Yaz, 2010a, 2010b; Wang, Yaz, \& Jeong, 2010; Wang, Yaz, \& Yaz, 2010, 2011).

Other possible performance criteria which can be used in this framework with various design parameters $\alpha, \beta, \gamma$ are given in Table 1. By satisfying the NLQR objective, the controller is designed to minimize the quadratic cost function. By satisfying the $H_{\infty}$ performance objective (Basar \& Bernhard, 1995; Van der Shaft, 1993), the synthesized controller achieves stabilization with robust disturbance suppression. By satisfying the passivity performance objective, the closed loop system is stable in an input-output sense (Khalil, 2002; Vidyasagar, 2002). 
Table 1. Various performance criteria in a general framework.

\begin{tabular}{llrl}
\hline$\alpha$ & $\beta$ & $\gamma$ & \multicolumn{1}{c}{ Performance criteria } \\
\hline 1 & 0 & $<0$ & Suboptimal NLQR- $H_{\infty}$ design \\
0 & 1 & 0 & NLQR-passivity design \\
0 & 1 & $>0$ & NLQR-input strict passivity design \\
$>0$ & 1 & 0 & NLQR-output strict passivity design \\
$>0$ & 1 & $>0$ & NLQR-very strict passivity \\
\hline
\end{tabular}

\section{Fuzzy LMI control with general performance criteria}

The following theorem summarizes the main results of the paper:

Theorem 1: Given the system model (10), performance output (11) and control input (9), if there exist matrices $S=$ $P^{-1}>0$ for all $t \geq 0$, such that the following $L M I$ holds:

$$
\left[\begin{array}{ccccc}
\Lambda_{11} & \Lambda_{12} & \Lambda_{13} & \Lambda_{14} & \Lambda_{15} \\
* & \Lambda_{22} & \Lambda_{23} & 0 & 0 \\
* & * & I & 0 & 0 \\
* & * & * & R^{-1} & 0 \\
* & * & * & * & I
\end{array}\right] \geq 0
$$

where

$$
\begin{aligned}
\Lambda_{11}= & -\frac{1}{2}\left[S A_{i}^{\top}-M_{j} B_{i}^{\top}+S A_{j}^{\top}-M_{i}^{\top} B_{j}^{\top}+A_{i} S-B_{i} M_{j}\right. \\
& \left.+A_{j} S-B_{j} M_{i}\right], \\
\Lambda_{12}= & -\frac{1}{2}\left(F_{i}+F_{j}\right)+\frac{\beta}{4}\left[S C_{i}^{\top}-M_{j}^{\top} D_{i}^{\top}+S C_{j}^{\top}-M_{i}^{\top} D_{j}^{\top}\right], \\
\Lambda_{13}= & \frac{1}{2} \alpha^{1 / 2}\left[S C_{i}^{\top}-M_{j}^{\top} D_{i}^{\top}+S C_{j}^{\top}-M_{i}^{\top} D_{j}^{\top}\right], \\
\Lambda_{14}= & \frac{1}{2}\left(M_{i}^{\top}+M_{j}^{\top}\right), \\
\Lambda_{15}= & S Q^{\top / 2} \\
\Lambda_{22}= & -\gamma I+\frac{1}{2} \beta \cdot\left(Z_{i}+Z_{j}\right)^{\top}, \\
\Lambda_{23}= & \frac{1}{2} \alpha^{1 / 2}\left[Z_{i}+Z_{j}\right]^{\top}
\end{aligned}
$$

using the notation

$$
M_{i}=K_{i} P^{-1}=K_{i} S
$$

then inequality (19) is satisfied.

Proof: By applying system models (10) and (14), performance outputs (11) and (15), and state feedback input (9), the performance index inequality (17) becomes

$$
\begin{aligned}
& {\left[\sum_{i=1}^{r} \sum_{j=1}^{r} h_{i}(\phi(t)) h_{j}(\phi(t)) \cdot G_{i j} \cdot x(t)\right.} \\
& \left.\quad+\sum_{i=1}^{r} h_{i}(\phi(t)) F_{i} w(t)\right]^{\top} \cdot P \cdot x(t)
\end{aligned}
$$

$$
\begin{aligned}
& +x^{\top}(t) \cdot P \cdot\left[\sum_{i=1}^{r} \sum_{j=1}^{r} h_{i}(\phi(t)) h_{j}(\phi(t)) \cdot G_{i j} \cdot x(t)\right. \\
& \left.+\sum_{i=1}^{r} h_{i}(\phi(t)) F_{i} w(t)\right]+x^{\top}(t) Q x(t) \\
& +\left[-\sum_{i=1}^{r} h_{i}(\phi(t)) K_{i} x(t)\right]^{\top} R\left[-\sum_{i=1}^{r} h_{i}(\phi(t)) K_{i} x(t)\right] \\
& +\alpha\left[\sum_{i=1}^{r} \sum_{j=1}^{r} h_{i}(\phi(t)) h_{j}(\phi(t)) \cdot H_{i j} \cdot x(t)\right. \\
& \left.+\sum_{i=1}^{r} h_{i}(\phi(t)) Z_{i} w(t)\right]^{\top} \\
& \cdot\left[\sum_{i=1}^{r} \sum_{j=1}^{r} h_{i}(\phi(t)) h_{j}(\phi(t)) \cdot H_{i j} \cdot x(t)\right. \\
& \left.+\sum_{i=1}^{r} h_{i}(\phi(t)) Z_{i} w(t)\right] \\
& -\beta\left[\sum_{i=1}^{r} \sum_{j=1}^{r} h_{i}(\phi(t)) h_{j}(\phi(t)) \cdot H_{i j} \cdot x(t)\right. \\
& \left.+\sum_{i=1}^{r} h_{i}(\phi(t)) Z_{i} w(t)\right]^{\top} \cdot w(t) \\
& +\gamma \cdot w^{\top}(t) w(t) \leq 0 .
\end{aligned}
$$

Inequality (23) is equivalent to

$$
\left[x^{\top}(t) \quad w^{\top}(t)\right]\left[\begin{array}{cc}
\Delta_{11} & \Delta_{12} \\
* & \Delta_{22}
\end{array}\right]\left[\begin{array}{l}
x(t) \\
w(t)
\end{array}\right] \leq 0
$$

where

$$
\begin{aligned}
\Delta_{11}= & \left(\sum_{i} \sum_{j} h_{i} h_{j} G_{i j}\right)^{\top} P+P\left(\sum_{i} \sum_{j} h_{i} h_{j} G_{i j}\right)+Q \\
& +\left[\sum_{i} h_{i} K_{i}\right]^{\top} R\left[\sum_{i} h_{i} K_{i}\right] \\
& +\alpha \cdot\left[\sum_{i} \sum_{j} h_{i} h_{j} H_{i j}\right]^{\top}\left[\sum_{i} \sum_{j} h_{i} h_{j} H_{i j}\right], \\
\Delta_{12}= & P\left(\sum_{i} h_{i} F_{i}\right)+\alpha \cdot\left[\sum_{i} \sum_{j} h_{i} h_{j} H_{i j}\right]^{\top}\left[\sum_{i} h_{i} Z_{i}\right] \\
& -\frac{\beta}{2} \cdot\left[\sum_{i} \sum_{j} h_{i} h_{j} H_{i j}\right]^{\top}
\end{aligned}
$$


$\Delta_{22}=\gamma l+\alpha \cdot\left[\sum_{i} h_{i} z_{i}\right]^{\top}\left[\sum_{i} h_{i} z_{i}\right]-\beta \cdot\left[\sum_{i} h_{i} z_{i}\right]^{\top}$.

Inequality (24) can be rewritten as

$$
\begin{gathered}
{\left[\begin{array}{cc}
\Psi_{11} & \Psi_{12} \\
* & \Psi_{22}
\end{array}\right]-\alpha \cdot\left[\begin{array}{c}
\left.\left[\sum_{i} \sum_{j} h_{i} h_{j} H_{i j}\right]^{\top}\right] \\
{\left[\sum_{i} h_{i} Z_{i}\right]^{\top}}
\end{array}\right]} \\
\times\left[\left[\sum_{i} \sum_{j} h_{i} h_{j} H_{i j}\right]\left[\sum_{i} h_{i} Z_{i}\right]\right] \geq 0,
\end{gathered}
$$

where

$$
\begin{aligned}
\Psi_{11}= & -\left(\sum_{i} \sum_{j} h_{i} h_{j} G_{i j}\right)^{\top} P-P\left(\sum_{i} \sum_{j} h_{i} h_{j} G_{i j}\right)-Q \\
& -\left[\sum_{i} h_{i} K_{i}\right]^{\top} R\left[\sum_{i} h_{i} K_{i}\right] \\
\Psi_{12}= & -P\left(\sum_{i} h_{i} F_{i}\right)+\frac{\beta}{2} \cdot\left[\sum_{i} \sum_{j} h_{i} h_{j} H_{i j}\right]^{\top} \\
\Psi_{22}= & -\gamma I+\beta \cdot\left[\sum_{i} h_{i} Z_{i}\right]^{\top} .
\end{aligned}
$$

By applying the Schur complement to inequality (26), we have

$$
\left[\begin{array}{ccc}
\Psi_{11} & \Psi_{12} & \alpha^{1 / 2}\left[\sum_{i} \sum_{j} h_{i} h_{j} H_{i j}\right]^{\top} \\
* & \Psi_{22} & \alpha^{1 / 2}\left[\sum_{i} h_{i} Z_{i}\right]^{\top} \\
* & * & l
\end{array}\right] \geq 0 .
$$

Similarly, inequality (28) can also be written as

$$
\begin{aligned}
& {\left[\begin{array}{ccc}
\Phi_{11} & \Phi_{12} & \alpha^{1 / 2}\left[\sum_{i} \sum_{j} h_{i} h_{j} H_{i j}\right]^{\top} \\
* & \Phi_{22} & \alpha^{1 / 2}\left[\sum_{i} h_{i} Z_{i}\right]^{\top} \\
* & * & l
\end{array}\right]-\left[\begin{array}{c}
\left.\sum_{i} h_{i} K_{i}\right]^{\top} \\
0 \\
0
\end{array}\right]} \\
& \times R\left[\left[\begin{array}{lll}
\left.\sum_{i} h_{i} K_{i}\right] & 0 & 0
\end{array}\right] \geq 0\right.
\end{aligned}
$$

where

$$
\begin{aligned}
& \Phi_{11}=-\left(\sum_{i} \sum_{j} h_{i} h_{j} G_{i j}\right)^{\top} P-P\left(\sum_{i} \sum_{j} h_{i} h_{j} G_{i j}\right)-Q \\
& \Phi_{12}=-P\left(\sum_{i} h_{i} F_{i}\right)+\frac{\beta}{2} \cdot\left[\sum_{i} \sum_{j} h_{i} h_{j} H_{i j}\right]^{\top} \\
& \Phi_{22}=-\gamma I+\beta \cdot\left[\sum_{i} h_{i} Z_{i}\right]^{\top} .
\end{aligned}
$$

By applying the Schur complement again to Equation (29), we have

$$
\left[\begin{array}{cccc}
\Phi_{11} & \Phi_{12} & \alpha^{1 / 2}\left(\sum_{i} \sum_{j} h_{i} h_{j} H_{i j}\right)^{\top} & {\left[\sum_{i} h_{i} K_{i}\right]^{\top}} \\
* & \Phi_{22} & \alpha^{1 / 2}\left[\sum_{i} h_{i} Z_{i}\right]^{\top} & 0 \\
* & * & I & 0 \\
* & * & * & R^{-1}
\end{array}\right] \geq 0 .
$$

Equivalently, we have

$$
\sum_{i} \sum_{j} h_{i} h_{j} \cdot\left[\begin{array}{cccc}
\Xi_{11} & \Xi_{12} & \Xi_{13} & \Xi_{14} \\
* & \Xi_{22} & \Xi_{23} & 0 \\
* & * & l & 0 \\
* & * & * & R^{-1}
\end{array}\right] \geq 0,
$$

where

$$
\begin{aligned}
\Xi_{11}= & -\frac{1}{2}\left[\left(A_{i}-B_{i} K_{j}\right)+\left(A_{j}-B_{j} K_{i}\right)\right]^{\top} \cdot P \\
& -\frac{1}{2} P \cdot\left[\left(A_{i}-B_{i} K_{j}\right)+\left(A_{j}-B_{j} K_{i}\right)\right]-Q \\
\Xi_{12}= & -\frac{1}{2} P\left(F_{i}+F_{j}\right)+\frac{\beta}{4}\left[\left(C_{i}-D_{i} K_{j}\right)+\left(C_{j}-D_{j} K_{i}\right)\right]^{\top} \\
\Xi_{13}= & \frac{1}{2} \alpha^{1 / 2}\left[\left(C_{i}-D_{i} K_{j}\right)+\left(C_{j}-D_{j} K_{i}\right)\right]^{\top} \\
\Xi_{14}= & \frac{1}{2}\left(K_{i}+K_{j}\right)^{\top} \\
\Xi_{22}= & -\gamma I+\frac{1}{2} \beta \cdot\left(Z_{i}+Z_{j}\right)^{\top} \\
\Xi_{23}= & \frac{1}{2} \alpha^{1 / 2}\left[Z_{i}+Z_{j}\right]^{\top} .
\end{aligned}
$$

Therefore, we have the following LMI

$$
\left[\begin{array}{cccc}
\Xi_{11} & \Xi_{12} & \Xi_{13} & \Xi_{14} \\
* & \Xi_{22} & \Xi_{23} & 0 \\
* & * & l & 0 \\
* & * & * & R^{-1}
\end{array}\right] \geq 0 .
$$

By multiplying both sides of the LMI above by the block diagonal matrix $\operatorname{diag}\{S, I, I, l\}$, where $S=P^{-1}$, and using 
the notation

$$
M_{i}=K_{i} P^{-1}=K_{i} S
$$

we obtain

$$
\left[\begin{array}{cccc}
\Theta_{11} & \Theta_{12} & \Theta_{13} & \Theta_{14} \\
* & \Theta_{22} & \Theta_{23} & 0 \\
* & * & I & 0 \\
* & * & * & R^{-1}
\end{array}\right] \geq 0
$$

where

$$
\begin{aligned}
\Theta_{11}= & -\frac{1}{2}\left[S A_{i}^{\top}-M_{j} B_{i}^{\top}+S A_{j}^{\top}-M_{i}^{\top} B_{j}^{\top}+A_{i} S-B_{i} M_{j}+A_{j} S\right. \\
& \left.-B_{j} M_{i}\right]-S Q S \\
\Theta_{12}= & -\frac{1}{2}\left(F_{i}+F_{j}\right)+\frac{\beta}{4}\left[S C_{i}^{\top}-M_{j}^{\top} D_{i}^{\top}+S C_{j}^{\top}-M_{i}^{\top} D_{j}^{\top}\right] \\
\Theta_{13}= & \frac{1}{2} \alpha^{1 / 2}\left[S C_{i}^{\top}-M_{j}^{\top} D_{i}^{\top}+S C_{j}^{\top}-M_{i}^{\top} D_{j}^{\top}\right] \\
\Theta_{14}= & \frac{1}{2}\left(M_{i}^{\top}+M_{j}^{\top}\right) \\
\Theta_{22}= & -\gamma I+\frac{1}{2} \beta \cdot\left(Z_{i}+Z_{j}\right)^{\top} \\
\Theta_{23}= & \frac{1}{2} \alpha^{1 / 2}\left[Z_{i}+Z_{j}\right]^{\top} .
\end{aligned}
$$

By applying the Schur complement again, the final LMI is derived

$$
\left[\begin{array}{ccccc}
\Lambda_{11} & \Lambda_{12} & \Lambda_{13} & \Lambda_{14} & \Lambda_{15} \\
* & \Lambda_{22} & \Lambda_{23} & 0 & 0 \\
* & * & I & 0 & 0 \\
* & * & * & R^{-1} & 0 \\
* & * & * & * & I
\end{array}\right] \geq 0,
$$

where

$$
\begin{aligned}
\Lambda_{11}= & -\frac{1}{2}\left[S A_{i}^{\top}-M_{j} B_{i}^{\top}+S A_{j}^{\top}-M_{i}^{\top} B_{j}^{\top}+A_{i} S-B_{i} M_{j}\right. \\
& \left.+A_{j} S-B_{j} M_{i}\right] \\
\Lambda_{12}= & -\frac{1}{2}\left(F_{i}+F_{j}\right)+\frac{\beta}{4}\left[S C_{i}^{\top}-M_{j}^{\top} D_{i}^{\top}+S C_{j}^{\top}-M_{i}^{\top} D_{j}^{\top}\right] \\
\Lambda_{13}= & \frac{1}{2} \alpha^{1 / 2}\left[S C_{i}^{\top}-M_{j}^{\top} D_{i}^{\top}+S C_{j}^{\top}-M_{i}^{\top} D_{j}^{\top}\right] \\
\Lambda_{14}= & \frac{1}{2}\left(M_{i}^{\top}+M_{j}^{\top}\right) \\
\Lambda_{15}= & S Q^{\top / 2} \\
\Lambda_{22}= & -\gamma^{I}+\frac{1}{2} \beta \cdot\left(Z_{i}+Z_{j}\right)^{\top} \\
\Lambda_{23}= & \frac{1}{2} \alpha^{1 / 2}\left[Z_{i}+Z_{j}\right]^{\top} .
\end{aligned}
$$

Hence, if LMI (38) holds, inequality (19) is satisfied. This concludes the proof of the theorem.
Remark 1: For the chosen performance criterion, LMI (38) needs to be solved each time to find matrices $S, M$; by using relation (18), we can find the feedback control gain. Therefore, the feedback control can be found to satisfy the chosen criterion.

\section{Application to the inverted pendulum on a cart}

The inverted pendulum on a cart problem is a benchmark control problem used widely to test control algorithms. A pendulum beam attached at one end can rotate freely in the vertical two-dimensional plane. The angle of the beam with respect to the vertical direction is denoted at angle $\theta$. The external force $u$ is desired to set the angle of the beam $\theta$ and angular velocity $\dot{\theta}$ to zero while satisfying the mixed performance criteria. A model of the inverted pendulum on a cart problem is given by Baumann \& Rugh (1986) and Tanaka \& Wang (2001):

$\dot{x}_{1}=x_{2}+\varepsilon_{1} \cdot w$

$\dot{x}_{2}=\frac{g \sin \left(x_{1}\right)-a m L x_{2}^{2} \cdot \sin \left(2 x_{1}\right) / 2-a \cos \left(x_{1}\right) u}{4 L / 3-a m L \cos ^{2}\left(x_{1}\right)}+\varepsilon_{2} \cdot w$,

where $x_{1}$ is the angle of the pendulum from the vertical direction; $x_{2}$ is the angular velocity of the pendulum; $g$ is the gravity constant; $m$ is the mass of the pendulum; is the mass of the cart; is the length to the pendulum centre of mass, length of the pendulum equals; is the external force, control input to the system; $w$ is the $L_{2}$ type of disturbance; $a$ is a constant, $a=1 /(m+M)$; and $\varepsilon_{1}, \varepsilon_{2}$ are the weighting coefficients of the disturbance.

Due to the system nonlinearity, we approximate the system using the following two-rule fuzzy model:

RULE 1:

IF $\left|x_{1}\right|$ is close to zero,

THEN $\dot{x}(t)=A_{1} x(t)+B_{1} u(t)+F_{1} w(t)$.

RULE 2:

IF $\left|x_{1}\right|$ is close to $\pi / 2$,

$\operatorname{THEN} \dot{x}(t)=A_{2} x(t)+B_{2} u(t)+F_{2} w(t)$.

where

$$
\begin{aligned}
& A_{1}=\left[\begin{array}{cc}
0 & 1 \\
\frac{g}{4 L / 3-a m L} & 0
\end{array}\right], \quad B_{1}=\left[\begin{array}{c}
0 \\
-\frac{a}{4 L / 3-a m L}
\end{array}\right], \\
& F_{1}=\left[\begin{array}{l}
\varepsilon_{1} \\
\varepsilon_{2}
\end{array}\right] \\
& A_{2}=\left[\begin{array}{cc}
0 & 1 \\
\frac{2 g}{\pi\left(4 L / 3-a m L \delta^{2}\right)} & 0
\end{array}\right],
\end{aligned}
$$




$$
\begin{aligned}
& B_{2}=\left[\begin{array}{c}
0 \\
-\frac{a \delta}{4 L / 3-a m L \delta^{2}}
\end{array}\right], \\
& F_{2}=\left[\begin{array}{l}
\varepsilon_{1} \\
\varepsilon_{2}
\end{array}\right] \quad \text { with } \delta=\cos \left(80^{\circ}\right) .
\end{aligned}
$$

The following values are used in our simulation:

$$
\begin{aligned}
& m=2 \mathrm{~kg}, \quad M=8 \mathrm{~kg}, \quad L=0.5 \mathrm{~m}, \quad g=9.8 \mathrm{~m} / \mathrm{s}^{2}, \\
& \varepsilon_{1}=1, \quad \varepsilon_{2}=0
\end{aligned}
$$

sampling time $T=0.001, x_{1}(0)=\pi / 6, x_{2}(0)=-\pi / 6$ as the initial conditions. The membership functions of Rules 1 and 2 are shown in Figure 1.

The following design parameters are chosen to satisfy:

Mixed NLQR- $H_{\infty}$ criteria: Mixed NLQR-passivity criteria: The mixed criteria control performance results are shown in Figures 2-4. From these figures, we find that the novel fuzzy LMI control has a satisfactory performance. The new technique controls the inverted pendulum very well under the effect of finite energy disturbance. It should also be noted that the LMI fuzzy control with mixed performance criteria satisfies global asymptotic stability.

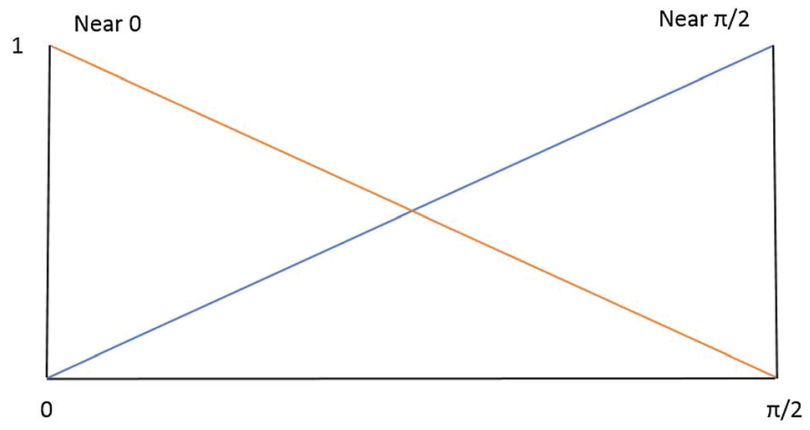

Figure 1. Membership functions of Rules 1 and 2.

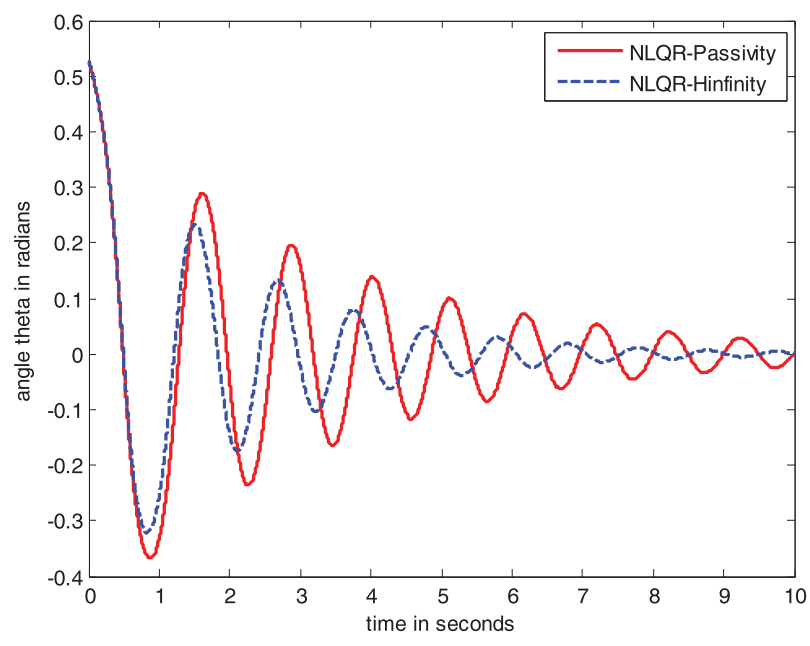

Figure 2. Angle trajectory of the inverted pendulum.

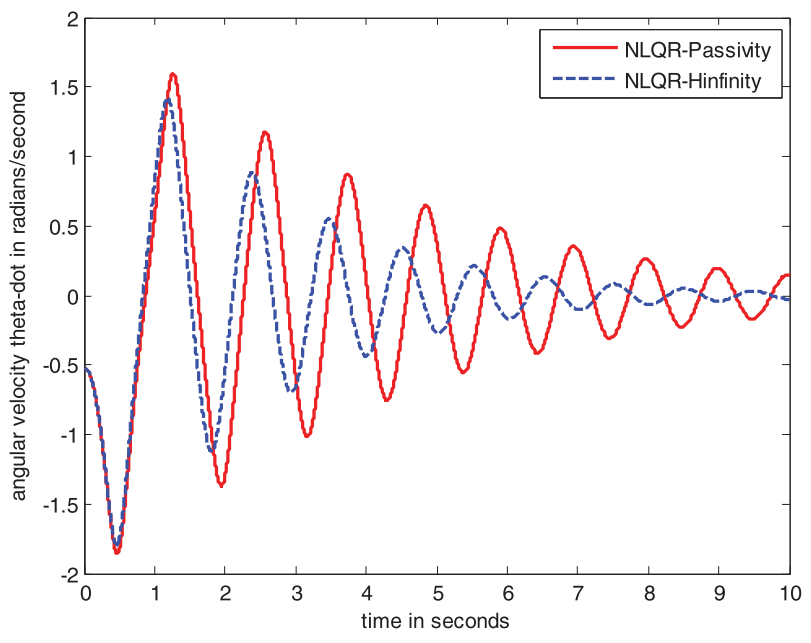

Figure 3. Angular velocity trajectory of the inverted pendulum.

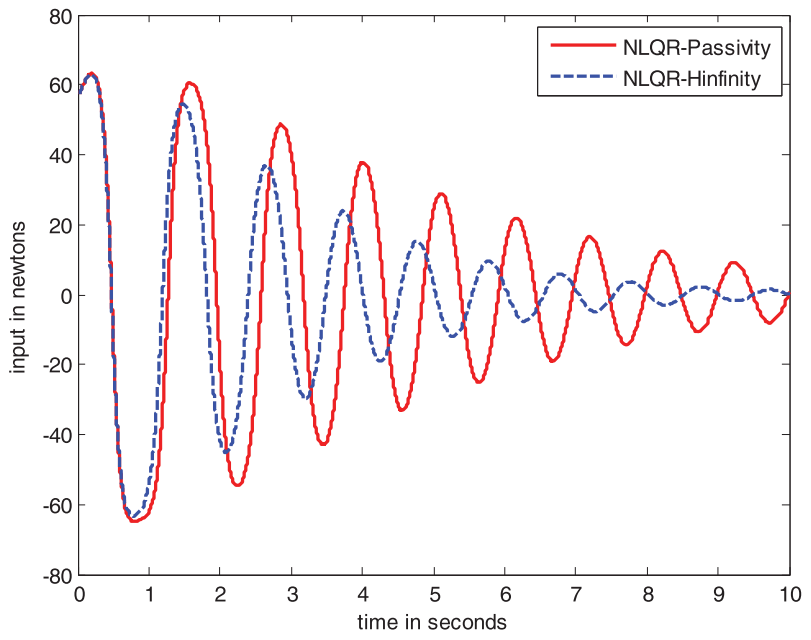

Figure 4. Control input applied to the inverted pendulum.

\section{Conclusions}

This paper presents a novel fuzzy control approach for continuous-time nonlinear systems based on LMI solutions. The T-S fuzzy model is applied to decompose the nonlinear system. Multiple performance criteria are used to design the controller and the relative weighting matrices of these criteria can be achieved by choosing different coefficient matrices. The optimal control can be obtained by solving the LMI at each time step. The inverted pendulum is used as an example to demonstrate its effectiveness. The simulation studies show that the proposed method provides a satisfactory alternative to the existing nonlinear control approaches.

\section{Disclosure statement}

No potential conflict of interest was reported by the authors. 


\section{References}

Basar, T., \& Bernhard, P. (1995). H-infinity optimal control and related minimax design problems - a dynamic game approach (2nd ed.). Boston, MA: Birkhauser.

Baumann, W. T., \& Rugh, W. J. (1986). Feedback control of nonlinear systems by extended linearization. IEEE Transactions on Automatic Control, 31(1), 40-46.

Boyd, S., Ghaoui, L. E., Feron, E., \& Balakrishnan, V. (1994). Linear matrix inequalities in system and control theory. SIAM Studies in Applied Mathematics. Philadelphia: SIAM.

Chen, B., Liu, X., Tong, S., \& Lin, C. (2007, October). Guaranteed cost control of T-S fuzzy systems with state and input delays. Fuzzy Sets and Systems, 158(20), 2251-2267.

Dong, J., Wang, Y., \& Yang, G. H. (2009, October). Control synthesis of continuous-time T-S fuzzy systems with local nonlinear models. IEEE Transactions on Systems, Man, and Cybernetics, Part B (Cybernetics), 39(5), 1245-1258.

Fang, C.-H., Liu, Y.-S., Kau, S.-W., Hong, L., \& Lee, C.-H. (2006, June). A new LMI based approach to relaxed quadratic stabilization of T-S fuzzy control systems. IEEE Transactions on Fuzzy Systems, 14(3), 386-397.

Feng, G. (2003, October). Controller synthesis of fuzzy dynamic systems based on piecewise Lyapunov functions. IEEE Transactions on Fuzzy Systems, 11(5), 605-612.

Gao, H., Liu, X., \& Lam, J. (2009). Stability analysis and stabilization for discrete-time fuzzy systems with time-varying delay. IEEE Transactions on Systems, Man, and Cybernetics, Part $B$ (Cybernetics), 39(2), 306-317.

Guerra, T. M., \& Vermeiren, L. (2004, May). LMI-based relaxed nonquadratic stabilization conditions for nonlinear systems in the Takagi-Sugeno's form. Automatica, 40(5), 823-829.

Huang, Y., \& Lu, W.-M. (1996). Nonlinear optimal control: Alternatives to Hamilton-Jacobi equation. Proc. of 35th conf. on decision and control, Kobe, Japan, pp. 3942-3947.

Johansson, M., Rantzer, A., \& Arzen, K. E. (1999, December). Piecewise quadratic stability of fuzzy systems. IEEE Transactions on Fuzzy Systems, 7(6), 713-722.

Khalil, H. K. (2002). Nonlinear systems (3rd ed.). Upper Saddle River, NJ: Prentice Hall.

Kim, E., \& Lee, H. (2000, October). New approaches to relaxed quadratic stability condition of fuzzy control systems. IEEE Transactions on Fuzzy Systems, 8(5), 523-534.

Kruszewski, A., Wang, R., \& Guerra, T. M. (2008, March). Nonquadratic stabilization conditions for a class of uncertain nonlinear discrete time TS fuzzy models: A new approach. IEEE Transactions on Automatic Control, 53(2), 606-611.

Lam, H. K., Leung, F. H. F., \& Tam, P. K. S. (2001). Fuzzy control of a class of multivariable nonlinear systems subject to parameter uncertainties: Model reference approach. International Journal of Approximate Reasoning, 26(2), 129-144.

Lam, H. K., Leung, F. H. F., \& Tam, P. K. S. (2002). A switching controller for uncertain nonlinear systems. IEEE Control Systems Magazine, 22(1), 7-14.

Lam, H. K., Li, H., \& Liu, H. (2013, March). Stability analysis and control synthesis for fuzzy-observer-based controller of nonlinear systems: A fuzzy-model-based control approach. IET Control Theory \& Applications, 7(5), 663-672.

Lee, K. R., Jeung, E. T., \& Park, H. B. (2001, May). Robust fuzzy $H_{\infty}$ control for uncertain nonlinear systems via state feedback: An LMI approach. Fuzzy Sets and Systems, 120(1), 123-134.

Lin, C., Wang, Q., \& Lee, T. (2005). Stabilization of uncertain fuzzy time-delay systems via variable structure control approach. IEEE Transactions on Fuzzy Systems, 13(6), 787-798.
Lin, C., Wang, Q., \& Lee, T. (2006). Delay-dependent LMI conditions for stability and stabilization of T-S fuzzy systems with bounded time-delay. Fuzzy Sets and Systems, 157(9), 1229-1247.

Liu, X., \& Zhang, Q. (2003, December). Approaches to quadratic stability conditions and $H_{\infty}$ control designs for T-S fuzzy systems. IEEE Transactions on Fuzzy Systems, 11(6), 830-839.

Lo, J.-C., \& Lin, M.-L. (2004, June). Observer-based robust $H_{\infty}$ control for fuzzy systems using two-step procedure. IEEE Transactions on Fuzzy Systems, 12(3), 350-359.

Lu, L. H., Huang, X., Gao, X. Z., Ban, X., \& Yin, H. (2007). Stability analysis of the simplest Takagi-Sugeno fuzzy control system using circle criterion. Journal of Systems Engineering and Electronics, 18(2), 311-319.

Mohseni, J., Yaz, E., \& Olejniczak, K. (1998). State dependent LMI control of discrete-time nonlinear systems. Proc. of the 37th IEEE conference on decision and control, Tampa, FL, pp. 4626-4627.

Nguang, S. K., \& Shi, P. (2006, August). Robust $H_{\infty}$ output feedback control design for fuzzy dynamic systems with quadratic D stability constraints: An LMI approach. Information Sciences, 176(15), 2161-2191.

Sala, A., \& Arino, C. (2007, December). Asymptotically necessary and sufficient conditions for stability and performance in fuzzy control: Applications of Polya's theorem. Fuzzy Sets and Systems, 158(24), 2671-2686.

Takagi, T., \& Sugeno, M. (1985). Fuzzy identification of systems and its applications to modeling and control. IEEE Transactions on Systems, Man, and Cybernetics, Part B (Cybernetics), 15 116-132.

Tanaka, K., Hori, T., \& Wang, H. O. (2003, August). A multiple Lyapunov function approach to stabilization of fuzzy control systems. IEEE Transactions on Fuzzy Systems, 11(4), 582-589.

Tanaka, K., Ikeda, T., \& Wang, H. O. (1996). Design of fuzzy control systems based on relaxed LMI stability conditions. 35th IEEE conference on decision and control, Kobe, Vol. 1, pp. 598-603.

Tanaka, K., \& Sugeno, M. (1990). Stability analysis of fuzzy systems using Lyapunov's direct method. Proc. NAFIPS'90, pp. 133-136.

Tanaka, K., \& Wang, H. O. (2001). Fuzzy control systems design and analysis - a linear matrix inequality approach. New York, NY: Wiley.

Teixeira, M. C. M., Assuncao, E., \& Avellar, R. G. (2003, October). On relaxed LMI based designs for fuzzy regulators and fuzzy observers. IEEE Transactions on Fuzzy Systems, 11(5), 613-623.

Teixeira, M. C. M., \& Zak, S. H. (1999, April). Stabilizing controller design for uncertain nonlinear systems using fuzzy models. IEEE Transactions on Fuzzy Systems, 7(2), 133-142.

Tong, S. C., He, X. L., \& Zhang, H. G. (2009). A combined backstepping and small-gain approach to robust adaptive fuzzy output feedback control. IEEE Transactions on Fuzzy Systems, 17(5), 1059-1069.

Tong, S. C., Liu, C., \& Li, Y. (2010). Fuzzy-adaptive decentralized output-feedback control for large-scale nonlinear systems with dynamical uncertainties. IEEE Transactions on Fuzzy Systems, 18(5), 845-861.

Tseng, C.-S., \& Hwang, C.-K. (2007, January). Fuzzy observerbased fuzzy control design for nonlinear systems with persistent bounded disturbances. Fuzzy Sets and Systems, 158(2), 164-179. 
Tuan, H. D., Apkarian, P., Narikiyo, T., \& Yamamoto, Y. (2001, April). Parameterized linear matrix inequality techniques in fuzzy control system design. IEEE Transactions on Fuzzy Systems, 9(2), 324-332.

Van der Shaft, A. J. (1993). Nonlinear state space $H_{\infty}$ control theory. In H. J. Trentelman \& J. C. Willems (Eds.), Perspectives in control. Boston, MA: Birkhauser.

Vidyasagar, M. (2002). Nonlinear system analysis (2nd ed.). Englewood Cliffs, NJ: SIAM.

Wang, L. X. (1994). Adaptive fuzzy systems and control: Design and stability analysis. Englewood Cliffs, NJ: Prentice Hall.

Wang, W.-J., Chen, Y.-J., \& Sun, C.-H. (2007, June). Relaxed stabilization criteria for discrete-time T-S fuzzy control systems based on a switching fuzzy model and piecewise Lyapunov function. IEEE Transactions on Systems, Man, and Cybernetics, Part B (Cybernetics), 37(3), 551-559.

Wang, W.-J., \& Sun, C.-H. (2005, December). Relaxed stability and stabilization conditions for a T-S fuzzy discrete system. Fuzzy Sets and Systems, 156(2), 208-225.

Wang, H. O., Tanaka, K., \& Griffin, M. (1996). An approach to fuzzy control of nonlinear systems: Stability and design issues. IEEE Transactions on Fuzzy Systems, 4(1), 14-23.

Wang, X., \& Yaz, E. E. (2010a, July). Robust multi-criteria optimal fuzzy control of continuous-time nonlinear systems. Proc. of the 2010 American control conference, Baltimore, MD, pp. 6460-6465.

Wang, X., \& Yaz, E. E. (2010b, December). Robust multi-criteria optimal fuzzy control of discrete-time nonlinear systems. Proc. of the 49th IEEE conference on decision and control, Atlanta, Georgia, pp. 4269-4274.

Wang, X., Yaz, E. E., \& Jeong, C. S. (2010, July). Robust nonlinear feedback control of discrete-time nonlinear systems with mixed performance criteria. Proc. of the 2010 American control conference, Baltimore, MD, pp. 6357-6362.

Wang, X., Yaz, E. E., \& Yaz, Y. I. (2010, December). Robust and resilient state dependent control of continuous-time nonlinear systems with general performance criteria. Proc. of the 49th IEEE conference on decision and control, Atlanta, Georgia, pp. 603-608.

Wang, X., Yaz, E. E., \& Yaz, Y. I. (2011). Robust and resilient state dependent control of discrete time nonlinear systems with general performance criteria. Proc. of the 18th IFAC congress, Milano, Italy, pp. 10904-10909.

Wu, H.-N. (2004, April). Reliable LQ fuzzy control for nonlinear discrete-time systems via LMIs. IEEE Transactions on Systems, Man, and Cybernetics, Part B (Cybernetics), 34(2), 1270-1275.

Wu, H.-N., \& Cai, K.-Y. (2004, December). H2 guaranteed cost fuzzy control for uncertain nonlinear systems via linear matrix inequalities. Fuzzy Sets and Systems, 148(3), 411-429. 